\title{
Coherent Schwinger Interaction from Darboux Transformation
}

\author{
Ekaterina Pozdeeva \\ Semenov Institute of Chemical Physics, \\ Russian Academy of Sciences *
}

\begin{abstract}
The exactly solvable scalar-tensor potential of the four-component Dirac equation has been obtained by the Darboux transformation method. The constructed potential has been interpreted in terms of nucleon-nuclear and Schwinger interactions of neutral particles with lattice sites during their channeling in the nonmagnetic crystal. The family of exactly solvable interaction Hamiltonians of a Schwinger type is obtained by means of the Darboux transformation chain. The analytic structure of the Lyapunov function of periodic continuation for each of the Hamiltonians of the family is considered.
\end{abstract}

\section{Introduction}

The Darboux (Moutard) transformation [1, 2, 3] is widely used for constructing solvable quantum mechanic models [4, 5, 6, 7]. It is known as the method of construction of the solvable potentials for the one-dimensional Schrödinger equation [8, 9, 10, 11] and one for two-component case was considered in [12. The quaternionic factorization of the Schrödinger equation was obtained in [13].

As was shown in [14, 15, 16, 17, 18, 19], the exactly solvable models for the one-dimensional Dirac equation with the Lorentz scalar potential employ the close relation between this equation and a pair of the Schrödinger equations. The solution of these equations, in turn, is based on well established algebraic techniques. In papers [20, 21, 22, 23] the Darboux transformation was applied directly to the onedimensional two-component Dirac equation, where as for applications it is often important the four-component Dirac equation [24, 25].

In works [26, 27] the attempts to use the Darboux transformation to the fourcomponent Dirac equation have been done. However, as a result, the Darboux transformation was applied to two-component equations.

In [13] the Darboux transformation of the Dirac equation was reduced to the quaternionic factorization of the Schrödinger equation. The Darboux - Crum method

*E-mail: ekatpozdeeva@mail.ru 
[1, 28] was applied to the Schrödinger equation in [29] and to the two-component Dirac equation in [21].

In the present work we apply the Darboux transformation to the one-dimensional four-component Dirac equation, thus we make contribution for applying the Darboux transformation to the full Dirac equation. Also we construct a chain of the Darboux - Crum transformations for the four-component Dirac equation. We try to generalize these results to construct an exactly solvable interaction Hamiltonian, which allows one to solve analytically the one-dimensional four-component Dirac equation.

The structure of the present paper is as follows. In section 2 we construct a Darboux transformation for the free Hamiltonian of the one-dimensional four-component Dirac equation and generate an exactly solvable transparent scalar-tensor potential. We show that the tensor part of the external potential has the meaning of the electrical strength. In section 3 we consider a some special choice of transformation parameters. In section 4 we construct a chain of the Darboux-Crum transformations for the four-component Dirac equation. In section 5 we briefly discuss the structure of the Lyapunov functions for the problem of the interaction with the periodic potentials. Finally, in section 6 we interpret the interaction of a spin- $1 / 2$ neutral massive particle with an external electrical field through its magnetic moment as coherent Schwinger interaction and try to apply these results to neutral particles channeling in crystals.

\section{Darboux transformation of the one-dimensional four-component Dirac equation}

The stationary one-dimensional Dirac equation reads:

$$
\begin{array}{r}
H \psi=E \psi, \quad H=H_{0}+H_{I}, \\
H_{0}=-i \alpha_{1} \partial_{x}+\beta m, \quad H_{I}=H_{I}(x),
\end{array}
$$

where $\alpha_{1}=\left(\begin{array}{cc}0 & \sigma_{1} \\ \sigma_{1} & 0\end{array}\right), \beta=\left(\begin{array}{cc}1_{2} & 0 \\ 0 & -1_{2}\end{array}\right), 1_{2}=\left(\begin{array}{cc}1 & 0 \\ 0 & 1\end{array}\right) ; \sigma_{1}$ is the Pauli matrix; $H_{0}$ is the free Dirac Hamiltonian [26, 27, 30, 31] and $H_{I}$ is the part of the Hamiltonian describing an interaction of the spin- $1 / 2$ particle with some external field. The solution of the equation (11) in the absence of the interaction is simple and well known for any $E$.

Let us consider the Darboux transformation

$$
H L=L H_{0}, \quad \psi=L \psi_{0}, \quad H \psi=E \psi
$$

with the operator $L$ of the form

$$
L=\partial_{x}-u_{x} u^{-1} .
$$

Here $4 \times 4$-matrix $u$ obeys the equation

$$
H_{0} u=u \Lambda,
$$


the $\Lambda$ is any nonsingular matrix, $u_{x}=d u / d x$.

Such transformation generates the interaction Hamiltonian

$$
H_{I}=H-H_{0}=-i\left(\alpha_{1} u_{x} u^{-1}-u_{x} u^{-1} \alpha_{1}\right) .
$$

If we verify that the function (2) is the solution of Eq. (1), where $H_{I}$ is defined by (5), we see hat $u$ is definite by (41).

We choose $\Lambda$ to be diagonal matrix of the form

$$
\Lambda=\left[\lambda_{1}(I+\beta)+\lambda_{2}(I-\beta)\right] / 2, \quad \lambda_{1,2} \leq m .
$$

Here $I=\left(\begin{array}{cc}1_{2} & 0 \\ 0 & 1_{2}\end{array}\right)$.

Let us construct the matrix $u$ :

$$
u=a I+b \alpha_{1}+c \beta+d \gamma, \quad \gamma=\alpha_{1} \beta,
$$

where

$$
\begin{gathered}
a=\left(\mu_{1}+\mu_{3}\right) / 2, \quad b=\left(\mu_{2}+\mu_{4}\right) / 2, \quad c=\left(\mu_{1}-\mu_{3}\right) / 2, \quad d=\left(\mu_{4}-\mu_{2}\right) / 2, \\
\mu_{1}=\cosh \left(k_{1} x+\phi_{1}\right), \quad \mu_{3}=\cosh \left(k_{2} x+\phi_{2}\right), \\
\mu_{2}=\frac{-i k_{2} \sinh \left(k_{2} x+\phi_{2}\right)}{\lambda_{2}-m}, \quad \mu_{4}=\frac{-i k_{1} \sinh \left(k_{1} x+\phi_{1}\right)}{\lambda_{1}+m}
\end{gathered}
$$

$k_{1,2}= \pm \sqrt{m^{2}-\lambda_{1,2}^{2}} ; \phi_{1,2}$ are arbitrary complex numbers. It is easy to check that these matrix is solution of the Eq. (4).

The inverse matrix reads:

$$
u^{-1}=\left[a I-b \alpha_{1}-c \beta-d \gamma\right] / D, \quad D=a^{2}-b^{2}-c^{2}+d^{2} .
$$

With the help of simple calculations it can be proved that

$$
H_{I}=H_{S}+H_{T}, \quad H_{S}=\beta V_{S}, \quad H_{T}=i \gamma V_{T} .
$$

Here $V_{S}, V_{T}$ are functions of $x$. The meaning of $V_{S}$ is transparent. It is an external scalar potential. The tensor part of the interaction Hamiltonian $H_{T}$ can be considered as particular case $\left(A_{\mu}=\left(A_{0}\left(x_{1}\right), \overrightarrow{0}\right)\right)$ of the more general tensor Hamiltonian

$$
\begin{aligned}
H_{T} & =g i \gamma_{0} \sigma_{\mu \nu} F_{\mu \nu}, \\
\sigma_{\mu \nu} & =\gamma_{\mu} \gamma_{\nu}-\gamma_{\nu} \gamma_{\mu}, \\
F_{\mu \nu} & =\frac{\partial A_{\nu}}{\partial x_{\mu}}-\frac{\partial A_{\mu}}{\partial x_{\nu}},
\end{aligned}
$$

where $\gamma_{\mu}$ are the Dirac matrices, $A_{\mu}$ is an external electromagnetic field and $g$ is a coupling constant proportional to magnetic moment of the particle.

Such Hamiltonian describes the interaction of a spin- $1 / 2$ neutral massive particle (e.g. neutron) with an external electrostatic field.

From above consideration we conclude that the tensor potential $V_{T}$ has meaning of the electrical strength

$$
V_{T} \sim E_{x}=-\frac{\partial A_{0}}{\partial x}
$$




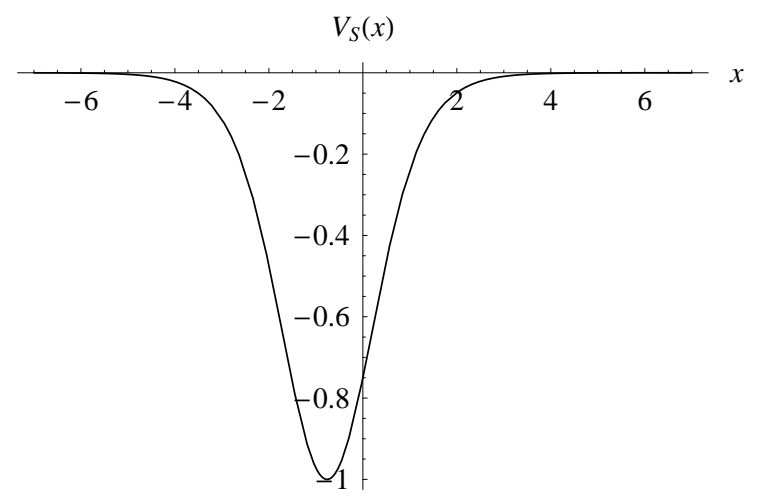

Figure 1: The scalar part of the external potential $V_{I}(x)=V_{S}(x)+V_{T}(x): V_{S}(x)=$ $-2 k^{2} /(m+\lambda \cosh (2 k x+2 \phi)), k=\sqrt{m^{2}-\lambda^{2}}, m=1, \lambda=1 / 2$.

\section{Some special choice of parameters}

Case 1. Let $\lambda_{1}=\lambda_{2}=\lambda, \phi_{1}=\phi_{2}=\phi$. In this case $V_{T}=0$

$$
V_{S}(x)=-\frac{2 k^{2}}{m+\lambda \cosh (2 k x+2 \phi)}, \quad k=\sqrt{m^{2}-\lambda^{2}} .
$$

This result coincides with the result of [32 at $\phi=[\ln (m+k) /(m-k)] / 4$.

The function $V_{S}(x)$ is shown for fixed values $m=1$ and $\lambda=1 / 2$ in Figure 1 ,

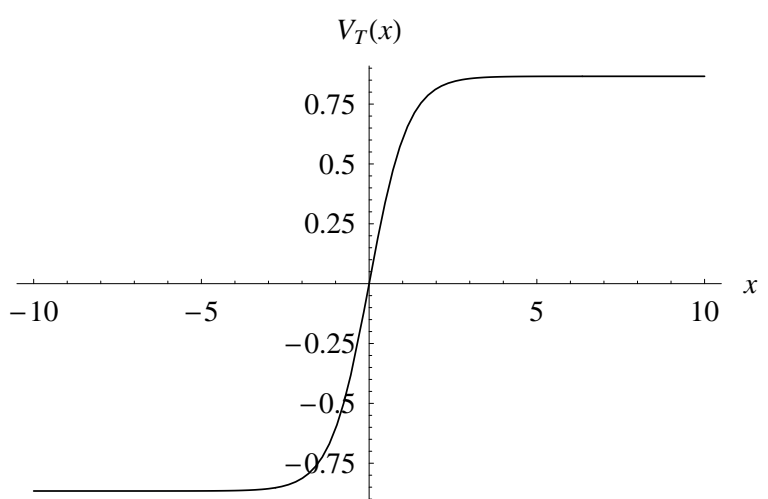

Figure 2: The tensor part of the $V_{I}(x): V_{T}(x)=k_{2} \tanh \left(k_{2} x\right), k_{2}=\sqrt{m^{2}-\lambda_{2}^{2}}$, $m=1, \lambda_{2}=1 / 2$.

Case 2. Let $\lambda_{1}=m, \phi_{1}=\phi_{2}=0$. In this case

$$
V_{S}=-\lambda_{2}-m=\text { const }
$$

which leads to the redefinition of the particle mass

$$
m \longrightarrow-\lambda_{2}
$$


The tensor potential (electrical strength) is

$$
V_{T}(x)=k_{2} \tanh \left(k_{2} x\right) \sim E_{x} .
$$

A pictorial representation of the potential $V_{T}(x)$ for fixed values $m=1$ and $\lambda_{2}=1 / 2$ is given in Figure 2,

Such electrical field is produced by the charge distribution

$$
\rho(x) \sim \frac{1}{\cosh ^{2}\left(k_{2} x\right)} .
$$

The charge distribution $\rho$ for values $m=1$ and $\lambda_{2}=1 / 2$ is shown in Figure 3 . It is easy to check that both $V_{S}$ and $V_{T}$ are transparent potentials.

\section{Simple chain of the Darboux-Crum transfor- mations}

As the next step consider a chain of the Darboux - Crum transformations for the four-component Dirac equation (11). Let us put in expressions (9), (10) $\phi_{1}=\phi_{2}=0$, $k_{1}=0, k_{2}=k, \lambda_{2}=\varepsilon_{1}=\sqrt{m^{2}-k^{2}}$. This leads to the Hamiltonian $H_{1}$ of the following form $H_{1}=-i \alpha_{1} \partial_{x}-\varepsilon_{1} \beta+i \gamma k \tanh (k x)$.

Consider the matrix

$$
u^{(1)}=a^{(1)} I+b^{(1)} \alpha_{1}+c^{(1)} \beta+d^{(1)} \gamma,
$$

where

$$
\begin{aligned}
& a^{(1)}=\left(\mu_{1}^{(1)}+\mu_{3}^{(1)}\right) / 2, \quad b^{(1)}=\left(\mu_{2}^{(1)}+\mu_{4}^{(1)}\right) / 2, \\
& c^{(1)}=\left(\mu_{1}^{(1)}-\mu_{3}^{(1)}\right) / 2, \quad d^{(1)}=\left(\mu_{4}^{(1)}-\mu_{2}^{(1)}\right) / 2, \\
& \mu_{1}^{(1)}=\cosh (k x), \quad \mu_{2}^{(1)}=\frac{-3 i k \sinh (k x) \cosh (k x)}{\varepsilon_{2}-\varepsilon_{1}}, \\
& \mu_{3}^{(1)}=\cosh ^{2}(k x), \quad \mu_{4}^{(1)}=0, \quad \varepsilon_{2}=\sqrt{m^{2}-4 k^{2}} .
\end{aligned}
$$

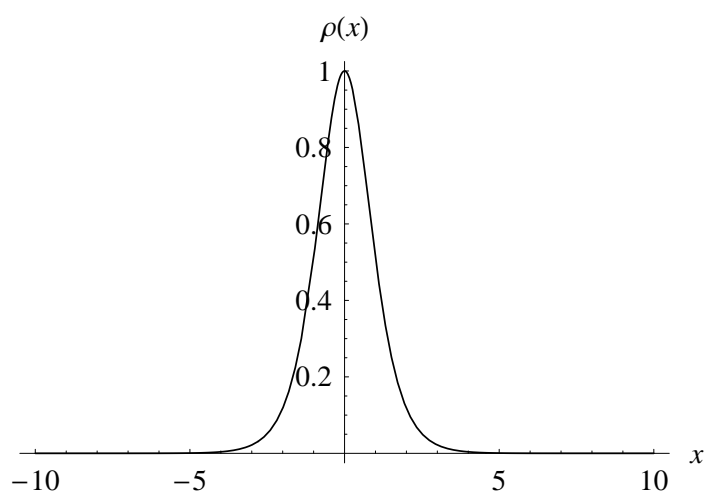

Figure 3: The charge distribution $\rho(x)=1 / \cosh ^{2}\left(k_{2} x\right), k_{2}=\sqrt{m^{2}-\lambda_{2}^{2}}, m=1$, $\lambda_{2}=1 / 2$. 
The matrix (22) obey the equation

$$
\begin{aligned}
H_{1} u_{1} & =u_{1} \Lambda_{1}, & \Lambda_{1} & =\frac{1}{2}\left[(I+\beta) \lambda_{1}^{(1)}+(I-\beta) \lambda_{2}^{(1)}\right], \\
\lambda_{1}^{(1)} & =-\varepsilon_{1}, & \lambda_{2}^{(1)} & =\varepsilon_{2} .
\end{aligned}
$$

Consider the intertwining relation

$$
L_{1} H_{1}=H_{2} L_{1}
$$

where

$$
L=\partial_{x}-u_{x}^{(1)} u^{(1)^{-1}}
$$

Then

$$
H_{2}-H_{1}=-i\left[\alpha_{1}, u_{x}^{(1)} u^{(1)^{-1}}\right]=\beta\left(\varepsilon_{1}-\varepsilon_{2}\right)+i \gamma k \tanh (k x)
$$

from which it follows

$$
H_{2}=-i \alpha_{1} \partial_{x}-\varepsilon_{2} \beta+i \gamma 2 k \tanh (k x) .
$$

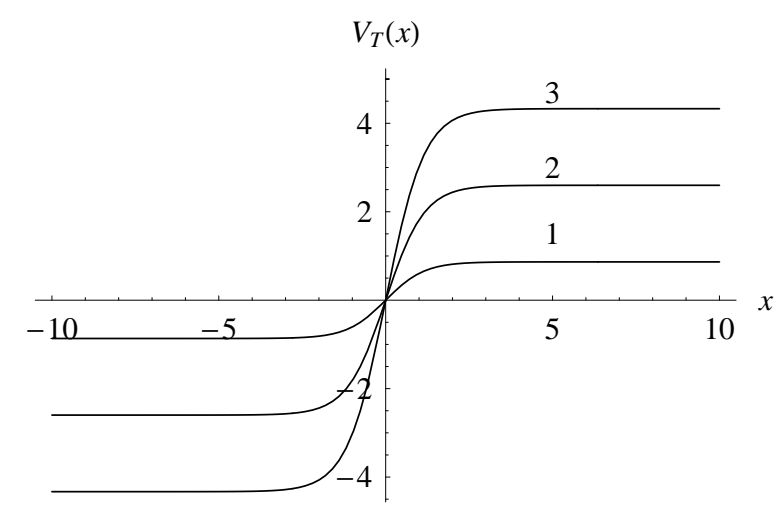

Figure 4: The tensor part of the interaction Hamiltonian (32): $V_{T}(x)=n k \tanh (k x)$, $k=\sqrt{m^{2}-\lambda_{2}^{2}}, m=1, \lambda_{2}=1 / 2($ line $1: n=1 ;$ line $2: n=3$; line $3: n=5$ )

Let

$$
H_{n}=-i \alpha_{1} \partial_{x}-\varepsilon_{n} \beta+i \gamma n k \tanh (k x), \quad \varepsilon_{n}=\sqrt{m^{2}-n^{2} k^{2}}
$$

and

$$
u^{(n)}=a^{(n)} I+b^{(n)} \alpha_{1}+c^{(n)} \beta+d^{(n)} \gamma
$$

where

$$
\begin{array}{ll}
a^{(n)}=\left(\mu_{1}^{(n)}+\mu_{3}^{(n)}\right) / 2, & b^{(n)}=\left(\mu_{2}^{(n)}+\mu_{4}^{(n)}\right) / 2, \\
c^{(n)}=\left(\mu_{1}^{(n)}-\mu_{3}^{(n)}\right) / 2, & d^{(n)}=\left(\mu_{4}^{(n)}-\mu_{2}^{(n)}\right) / 2,
\end{array}
$$




$$
\begin{aligned}
& \mu_{1}^{(n)}=\cosh ^{n}(k x), \quad \mu_{2}^{(1)}=\frac{-i(2 n+1) k \sinh (k x) \cosh ^{(n-1)}(k x)}{\varepsilon_{n+1}-\varepsilon_{n}} \\
& \mu_{3}^{(1)}=\cosh ^{(n+1)}(k x), \quad \mu_{4}^{(1)}=0 .
\end{aligned}
$$

Then $u_{n}$ obey the equation

$$
\begin{aligned}
H_{n} u_{n} & =u_{n} \Lambda_{n}, \quad \Lambda_{n}=\frac{1}{2}\left[(I+\beta) \lambda_{1}^{(n)}+(I-\beta) \lambda_{2}^{(n)}\right], \\
\lambda_{1}^{(n)} & =-\varepsilon_{n}, \quad \lambda^{(n)}=\varepsilon_{n+1} .
\end{aligned}
$$

The solutions of the equation

$$
H_{n+1} \psi_{s}=E_{s} \psi_{s}
$$

corresponding the discrete levels are of the following form:

$$
\begin{aligned}
\phi_{s} & =\left(\phi_{s 1}, \phi_{s 2}, \phi_{s 3}, \phi_{s 4}\right)^{t}, \\
\phi_{s 1} & =A P_{n}^{s}\{\tanh (k x)\}, \quad \phi_{s 2}=B P_{n}^{s}\{\tanh (k x)\} \\
\phi_{s 3} & =C P_{n+1}^{s}\{\tanh (k x)\}, \quad \phi_{s 4}=D P_{n+1}^{s}\{\tanh (k x)\} \\
\frac{A}{D} & =\frac{B}{C}=\frac{k(n+s+1)}{\varepsilon_{n+1}+E_{s}} \\
E_{s} & =\operatorname{sign}(s) \varepsilon_{s}, \quad \varepsilon_{s}=\sqrt{m^{2}-s^{2} k^{2}} \\
s & =-n,-n+1, \ldots,-2,-1,1,2, \ldots, n, n+1,
\end{aligned}
$$

where $P_{n}^{s}$ are associated Legendre polynomials [33], $D$ and $C$ are arbitrary nonzero numbers.

The solutions of the equation

$$
H_{n+1} \psi=E \psi, \quad|E|>m
$$

corresponding to the continuous spectrum can be obtained from (40) by the substitution

$$
\begin{aligned}
& P_{n(n+1)}^{s}\{\tanh (k x)\} \Rightarrow P_{n(n+1)}^{\mu}\{\tanh (k x)\}, \quad \mu= \pm i p / k, \quad p=\sqrt{E^{2}-m^{2}}, \\
& P_{n}^{( \pm i p / k)}\{\tanh (k x)\}=\frac{\exp ( \pm i p x)}{\Gamma(1 \mp i p / k)} \cdot F\left(-n ; n+1 ; 1 \mp \frac{i p}{k} ; \frac{1-\tanh (k x)}{2}\right) .
\end{aligned}
$$

From the last expression it is evident that "potentials" of the form $-\beta \varepsilon_{n}+i \gamma n \tanh (k x)$ are transparent.

\section{Exactly solvable periodic potentials}

Starting with exactly solvable potential for full real axis one can construct the exactly solvable periodic potentials in the manner described in the papers [10, 22, 34]. 
It is well known that the continuous spectrum of such potentials have a band structure. For the investigation of this structure it is necessary to construct so-called Lyapunov function $D(E)$ (see for details [10, 22, 34]).

The values of energy $E$ which obey the equation $|D(E)|>2$ belong to the forbidden bands. All others belong to the allowed bands.

Omitting simple but cumbersome calculations we present here only final result for the some lowest values of the $n$ :

$$
\begin{gathered}
D_{n}(E)=2\left[\cos (2 p a) A_{n}\left(p, t_{1}\right)-\left(k t_{1} / p\right) \sin (2 p a)\right] B_{n}\left(p, t_{1}\right), \\
t_{1}=\tanh (k a), \quad a=T / 2
\end{gathered}
$$

where $T$ is the parameter of periodization [10, 22, 34]. In general case (of the arbitrary $n$ )

$$
\begin{gathered}
A_{n}=\frac{\operatorname{Im} \Phi_{n}\left(\nu, t_{1}\right)}{\nu}, \quad B_{n}=\frac{\operatorname{Re} \Phi_{n}\left(\nu, t_{1}\right)}{t_{1}}, \\
\Phi_{n}=(n-i \nu) \cdot F\left(-n+1, n ; 1-i \nu ; \frac{1-t_{1}}{2}\right) \cdot F\left(-n, n+1 ; 1+i \nu ; \frac{1+t_{1}}{2}\right), \\
\nu=p / k .
\end{gathered}
$$

In the case $\left.k a>>1\left(1-t_{1}<<1\right) 1\right)$, it is readily found from (??) and (??)

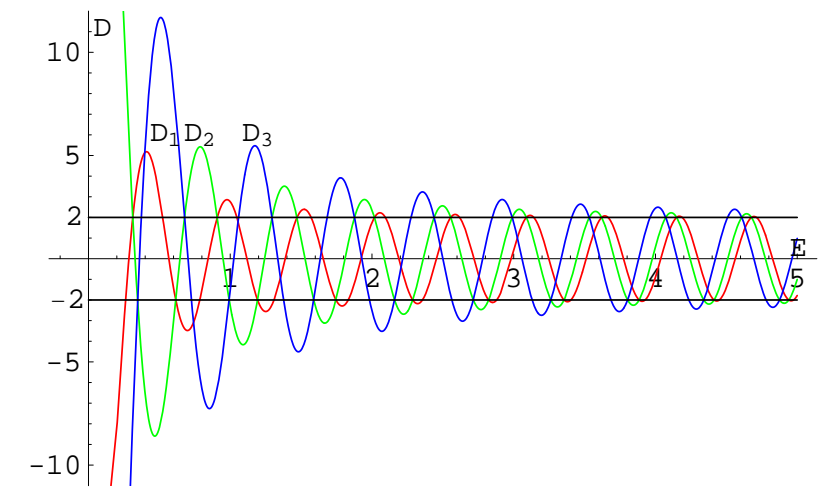

Figure 5: Lyapunov functions $D_{n}, n=\overline{1,3}$, with $k a=6$.

$$
\begin{gathered}
D_{n}(E)=2 \frac{\cos \left(\phi_{n}-\arctan (n k / p)\right)}{\cos (\arctan (n k / p))}, \\
\phi_{n}=2\left[p a+\sum_{s=1}^{n} \arctan (s k / p)\right] .
\end{gathered}
$$

The solution of inequality $\left|D_{n}(E)\right| \geq 2$ is trivial in this case.

Lyapunov functions $D_{n}(E)$ with $n=\overline{1,3}$ are presented in Fig. 5 for $k a=6$. 


\section{Discussion}

We show that the Darboux transformation for the free one-dimensional four-component Dirac Hamiltonian generates the interaction that can takes place in the real world.

The scalar potential arises in the theory of nuclear-nuclear interactions [35, 36] when sigma-exchange is taken into account [37, 38] and can be considered as a part of the nuclear potential in the channeling theory [39, 40].

The interaction of a neutral massive particle with the spin $1 / 2$ (e.g.neutron) with an external electrostatic field generated by the Darboux transformation can be interpreted as Schwinger interaction of neutron with atoms of the lattice in its channeling in the nonmagnetic crystals [39, 41, 42, 43, 44, 45, 46].

The origin of the Schwinger interaction is due to relativistic effect of appearance of the lattice magnetic field in the neutron rest frame, when it passes through the electrical field of the crystal. Due to proper magnetic moment of neutron the appearance of such field leads to magnetic interaction, which allows to order and orientate movement of neutron as in the case of channeling in nonmagnetic crystal [39, 40, 47, 48, 49].

Really, in the coordinate system connected with the neutron moving in the electrical field of the crystal the magnetic field $\vec{H}=[\vec{E}, \vec{v}]$ appears. In the case of the plane channel the value of the $\vec{E}$ averaged by the area of elementary cell goes to zero. As a result, the mean value of the $\vec{H}$ obtains its maximum near the plane. In the middle of two planes $\vec{H}$ goes to zero, changes the sign and increases to maximum value near the next plane of the channel (see Figures 2 and 4).

Moving across the plane the effective field $\vec{H}$, due to the changing of the sign of the electrical field vector $\vec{E}$, change the sign and its space behaviour repeating the behaviour in the previous channel (Figure 5).

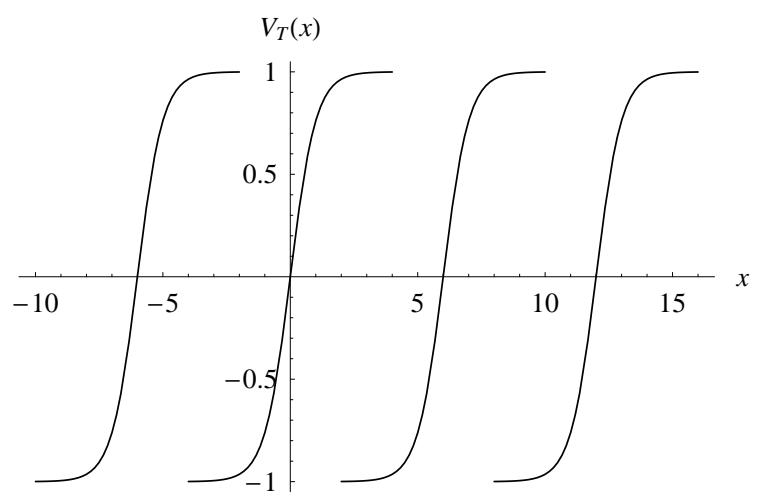

Figure 6: Periodic continuation of the potential (20) for $k_{2}=1$.

In accordance with the accepted terminology, the considered neutron-cell interaction may be characterized as coherent Schwinger interaction and is the generalization of well known neutron-atom Schwinger interaction [39]. 


\section{Acknowledgments}

The author is grateful to Prof. A. V. Tarasov for fruitful discussions and Dr. S. R. Gevorkyan who have read the paper and made useful comments. I am grateful to Joint Institute for Nuclear Research (Dubna, Moscow region, Russia) for hospitality during this work. The work was supported in part by the grant of Dinastiya Foundation for Noncommercial Programs and Moscow International Center of Fundamental Physics.

\section{References}

[1] G. Darboux, C. R. Acad. Sci., Paris 941456 (1882) arXiv:physics/9908003.

[2] Th. F. Moutard, C. R. Acad. Sci., Paris 80729 (1875).

[3] H. C. Rosu, Short Survey of Darboux Transformations, arXiv:quant-ph/980956.

[4] M. J. Ablowitz and H. Segur, Solitons and the Inverse Scattering Transforms (Philadelpia: SIAM, 1981).

[5] V. B. Matveev and M. A. Salle, Darboux Transformations and Solitons (Berlin: Springer, 1991).

[6] V. G. Bagrov and B. F. Samsonov, Phys. Part. Nucl., 28374 (1997).

[7] A. M. Pupasov and B. F. Samsonov, Symmetry, Integrability and Geometry: Methods and Applications, 1020 (2005).

[8] C. V. Sukumar, J. Phys., A 182917 (1985).

[9] V. G. Bagrov and B. F. Samsonov, Theor. Math. Phys., 104356 (1995).

[10] B. F. Samsonov, Eur. J. Phys., 201305 (2001).

[11] A. A. Suzko, Int. J. Mod. Phys., A 12277 (1997).

[12] A. A. Suzko, Phys. Lett., A 308267 (2003).

[13] V. G. Kravchenko and V. V. Kravchenko, On a generalized Schrödinger operator and Darboux transformation, in: T. E. Simos (Ed.), Proc. Intern. Conf. Num. Analys. Appl. Math., Chalkis, Greece, 2004 (Weinheim: Wiley-VCH, 502-505, 2004).

[14] F. Cooper, A. Khare, R. Musto et al., Ann. Phys., A 1871 (1988).

[15] G. J. Clerk and B. H. J. McKellar, Phys. Rev., B 476942 (1993).

[16] F. A. B. Coitinho and Y. Nogami, Phys. Lett., A 124211 (1987).

[17] U. Percoco and V. M. Villalba, Phys. Lett., A 141211 (1989). 
[18] Y. Nogami and F. M. Toyama, Phys. Rev., A 471708 (1993).

[19] C. L. Roy, Phys. Lett., A 189345 (1994).

[20] A. A. Stahlhofen, J. Phys., A 278379 (1994).

[21] L. M. Nieto, A. A. Pecheritsin and B. F. Samsonov, Ann. Phys., 305151 (2003).

[22] B. F. Samsonov, A. A. Pecheritsin, E. O. Pozdeeva et al., Eur. J. Phys., 24 435 (2003).

[23] A. A. Pecheritsin, E. O. Pozdeeva, B. F. Samsonov. Russ. Phys. J., 48365 (2005).

[24] Proceedings Intern. PCI-Conference (Moscow: SNPRI, 2006).

[25] Theory of radiation of relativistic particles, Ed. by V. A. Bordovitsin (Moscow: Physmathlit, 2002).

[26] A. Anderson, Phys. Rev., D 37536 (1988).

[27] A. V. Yurov, Phys. Lett., A 22551 (1987).

[28] M. M. Crum, Q.J.Math., Oxford, 6121 (1955) arXiv:physics/9908019.

[29] A. A. Andrianov and F. Cannata, J. Phys., A 3710297 (2004).

[30] E. O. Pozdeeva, J. Surf. Invest., 366 (2007).

[31] B. Thaller, The Dirac Equation (Berlin: Springer, 1992).

[32] Y. Nogami and F. M. Toyama, Phys. Rev., A 5793 (1998).

[33] I. S. Gradshtein and I. M. Ryzhik, Tables of Integrals, Series and Products (Moscow: Nauka Publication, 1971).

[34] V. G. Bagrov, A. A. Pecheritsin, E. O. Pozdeeva et al., Commun. Nonlin. Scien. Num. Simulat., 913 (2004).

[35] J. Sucher, The concept of potential in quantum field theory, arXiv:hep-ph/9412388.

[36] V. K. Ignatovich, Usp. Fiz. Nauk, 150144 (1986).

[37] F. Partovi and E. L. Lomon, Phys. Rev., D 51192 (1972).

[38] J. F. Donoghue, Phys. Lett., B 643165 (2006).

[39] V. I. Vysotskii and R. N. Kuzmin, Usp. Fiz. Nauk, 1621 (1992).

[40] V. V. Fedorov and V. V. Voronin, Dynamic diffraction and optics of neutrons in noncentrosymmetrical crystals. Search of EDM of neutron: new possibilities (Saint-Petersburg: PINP, 2004). 
[41] A. G. Kadmenskii, V. V. Samarin and A. F. Tulinov, Phys. Part. Nucl., 34823 (2003).

[42] Channeling: theory, observation and application, Ed. by D.V. Morgan (London: Academic Press, 1973).

[43] V. A. Ryabov, Channeling effect (M.: Energoizdat, 1994).

[44] Proc. Int. Conf. on Charged and Neutral Particle Channeling "Channeling 2004" (November 2004, Frascati), Proc. of SPIE V. 5974, 2005.

[45] Proc. Int. Conf. "Channeling 2005", J. Rus. Laser Res., 25 (2006).

[46] Proc. Int. Conf. on Charged and Neutral Particle Channeling "Channeling 2006"-http://www.lnf.infn.it/conference/channeling2006

[47] V. V. Fedorov, E. G. Lapin and S. Y. Semenikhin, Appl. Phys., A 74 [Suppl. 1] 91 (2002).

[48] F. L. Shapiro, Usp. Fiz. Nauk, 95145 (1968).

[49] N. A. Vlasov, Neutrons (M.: Nauka, 1973). 